\title{
A STUDY ON SEISMIC ANALYSIS METHODS IN THE CROSS SECTION OF UNDERGROUND STRUCTURES USING STATIC FINITE ELEMENT METHOD
}

\author{
Akira TATEISHI ${ }^{1}$ \\ ${ }^{1}$ Member of JSCE, Dr. Eng., Civil Engineering Research Institute, TAISEI CORPORATION \\ (344-1, Nase-cho, Totsuka-ku, Yokohama 245-0051, Japan)
}

\begin{abstract}
Based on the dynamic substructure technique, the author proposes a static seismic analysis method, the Ground Response Method, for underground structures modeled on the structure and surrounding soil that is expressed using finite elements. By comparing existing static seismic analysis methods, it is verified that the Ground Response Acceleration Method for Buried Structures provides results almost equivalent to those obtained from the Ground Response Method. However, the FEM Seismic Deformation Method yields different results. Thus, the author proposes a new seismic load that is equivalent to seismic ground strains and modifies the FEM Seismic Deformation Method.
\end{abstract}

Key Words : underground structure, seismic design, substructure method, static finite element method, seismic load

\section{OUTLINE}

The dynamic behavior of underground structures has been demonstrated through verification studies such as the several earthquake observations and model vibration experiments. Based on the results, the characteristics of this behavior of underground structures have been identified as:

a) Underground structures have no predominant vibration modes themselves and are dependent on the surrounding soil.

b) The deformation of underground structures is controlled by relative displacements or strains of the surrounding soil.

A few static seismic analysis methods for underground structures have been proposed based on the

This paper is translated into English from the Japanese paper, which originally appeared on J. Struct. Mech. Earthquake Eng., JSCE, No.519/I-32, pp.139148, 1995.7. above knowledge and these can be classified into two groups in terms of modeling techniques for the surrounding soil. One of these techniques is of soilspring type, in which the surrounding soil is modeled using springs; the representative method is the seismic deformation method. The seismic deformation method has been primarily adapted to the seismic design of slender underground structures in the longitudinal direction, such as that of a buried pipe, as described in Establishment of New Aseismic Design Method ${ }^{1)}$. In 1985, the method was modified for the seismic design of underground structures in the transverse direction of nuclear power plants and its applicability was shown ${ }^{2}$. The advantages of the methods of the soil-spring type are that the modeling is relatively easy and that the resulting stresses of underground structures can be expressed by simple equations. Hence, this type is widely used for the seismic design of underground structures. The other type is the soil-FEM type, in which the surrounding soil is modeled with finite elements. One 
of the representative methods of this type is the seismic deformation method proposed by Hamada et al. ${ }^{3), 4)}$, which is used for design of underground rock caverns in the transverse direction. The other is Ground Response Acceleration Method for Buried Structures (GRAMBS) proposed by Katayamas), 6). In this paper, the seismic deformation method of the soil-FEM type is called the FEM Seismic Deformation Method (FSDM) in order to distinguish it from the seismic deformation method of the soil-spring type. The advantages of the soil-FEM type are that the calculation of soil-spring constants is not necessary and that the seismic stability of surrounding soil can be obtained using the resulting stresses of FEM elements of soil as well as those of underground structures.

In general, the dynamic substructure method ${ }^{7)}$ is one of the most effective methods for analyzing dynamic interaction behavior of a finite region such as a structure or the soil surrounding the structure, which, in reality, is continuous in a half-space. With regard to the static seismic analysis method of the soil-spring type, the author studied a seismic load for the seismic deformation method based on the dynamic substructure method and verified that seismic stresses and seismic deformation of soil should be applied to an underground structure. The author then proposed the modified method ${ }^{8)}$.

On the other hand, with regard to the static seismic analysis method of the soil-FEM type, Hibino et al. conducted dynamic FEM analyses and static FEM analyses using FSDM and GRAMBS for a large underground rock cavern. The results showed that the maximum stresses of the concrete lining of the cavern obtained using GRAMBS were in good agreement with those obtained using the dynamic FEM; however, the stresses obtained using FSDM were not in good agreement ${ }^{9}$. There is a big difference between FSDM and GRAMBS in terms of seismic load application; the former is a seismic displacement of soil and the latter is an inertia force of soil respectively. It can be seen that there remains an unsolved problem in the static seismic analysis method of the soil-FEM type. Therefore, the author attempts to theoretically demonstrate the seismic load application of a static seismic analysis method of the soil-FEM type based on the dynamic substructure method.

Following is an outline of the paper. First, existing static seismic analysis methods of the soil-FEM type are introduced, and then comparative analyses of an underground duct in the transverse direction are conducted using the existing methods, in order to examine the unsolved problem. Second, a static substructure method of the soil-FEM type for an under- ground structure based on the dynamic substructure method is proposed. Through a comparison between the seismic loads of the static substructure method and the existing methods, the author proposes a new method of seismic load application and verifies the validity through numerical analyses of the underground duct. Finally, characteristics of the existing methods and the methods proposed in this paper are compared.

\section{STATIC SEISMIC ANALYSIS METH- ODS OF THE SOIL-FEM TYPE}

\section{(1) FEM seismic deformation method}

Hamada et al. ${ }^{3), 4)}$ demonstrated that the seismic strains of the surrounding soil was statically transferred to an underground rock cavern according to the record of earthquake motions at a mountain tunnel. This phenomenon had been already introduced in a seismic design method of a tunnel structure in the longitudinal direction. Based on these findings, FSDM was proposed. In this method, the maximum seismic strains of soil are calculated, the underground structure is not modeled (termed as a free field), and then the relative displacements obtained from the calculated strains are applied to the outer boundaries of an FEM model that expresses the surrounding soil of an underground rock cavern. The surrounding soil is modeled with finite elements in order to study the seismic stability of the surrounding soil as well as the tunnel structure. A conceptual figure of a seismic load of FSDM is shown in Fig. 1.

\section{(2) Ground response acceleration method for buried structures}

Katayama ${ }^{5), 6)}$ insisted that existing empirical calculation methods of a subgrade reaction spring for the seismic deformation method didn't have enough accuracy. Hence, in place of the seismic deformation method, he proposed GRAMBS based on the characteristics of the dynamic behavior of underground structures. In this method, the surrounding soil is modeled by means of finite elements and accelerations are calculated in a free field. The calculated accelerations are then applied to the FEM model, including an underground structure, in which horizontal rollers are set at side boundaries in the case of horizontal earthquake motions. A conceptual figure of a seismic load of GRAMBS is shown in Fig. 2. This method has been adapted to the seismic design of underground ducts, shafts, and rock caverns. 


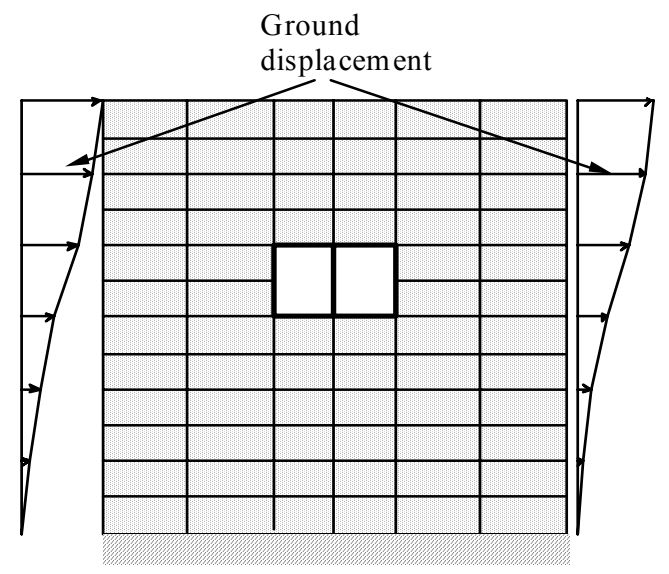

Fig. 1 Seismic load of FSDM

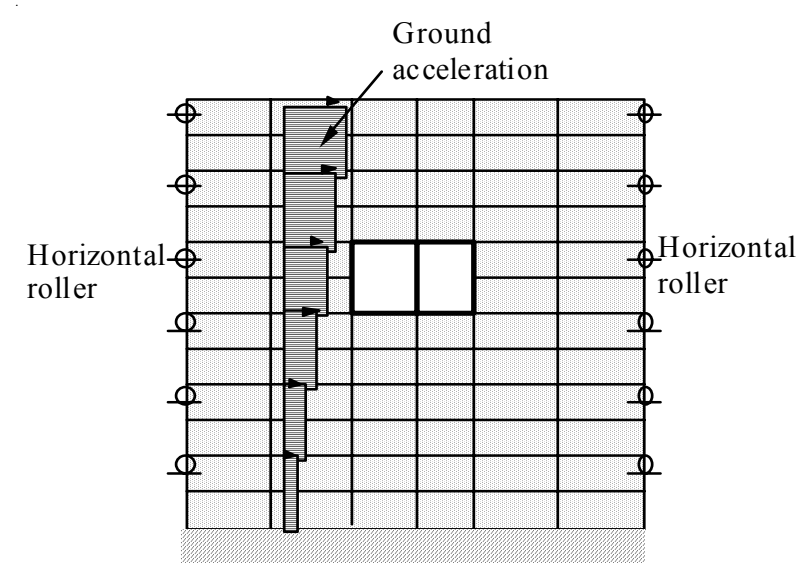

Fig. 2 Seismic load of GRAMBS

\section{COMPARATIVE STUDY THROUGH NUMERICAL ANALYSES}

In order to identify problems in existing static seismic analysis methods of the soil-FEM type, numerical analyses by means of a dynamic FEM, FSDM, and GRAMBS were conducted using an underground duct in the transverse direction as an object of study. The maximum seismic section forces obtained from the numerical analyses were then compared.

\section{(1) Numerical conditions}

The structure, used as the object of study, is a two-box-type reinforced concrete duct, which is buried in a sandy layer. The ground consists of a sandy layer and a rocky layer. Two kinds of numerical models are prepared; one model is a covered duct and the other model is an uncovered duct. The

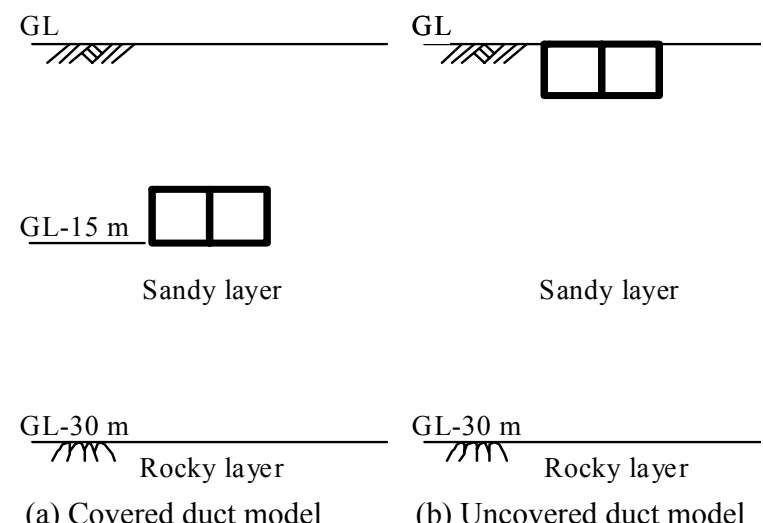

(a) Covered duct model

(b) Uncovered duct model

Fig. 3 Ground and duct model

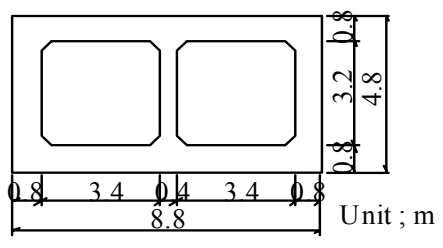

Fig. 4 Dimensions of duct model

model of the ground and the duct is shown in Fig. 3 and the dimensions of the duct are shown in Fig. 4.

The duct is modeled using a linear elastic beam element. The physical properties of the duct material are shown in Table 1. In the case of the sandy layer, an effective overburden stress dependency of modulus and the strain dependency of modulus reduction and damping are taken into consideration. The rocky layer is modeled using a linear elastic material. The physical properties of the soil are shown in Table 2.

The input earthquake motion is the acceleration time history of the EL CENTRO NS-component whose maximum acceleration is normalized to 300 $\mathrm{cm} / \mathrm{sec}^{2}$; the acceleration time history is input to the rock surface at a depth of GL-30m as a shear wave.

\section{(2) Numerical methods}

First, one-dimensional site response analysis based on the multi-reflection theory for layered soil (termed as the multi-reflection analysis) is conducted using the equivalent linear technique. Then, the site responses as a free field are calculated and the equivalent seismic rigidity and damping of the layered soil are obtained. The equivalent seismic rigidity is used in every seismic analysis hereafter.

Second, two-dimensional dynamic FEM based on the complex response analysis method is conducted. With regard to boundary conditions, an energytransmitting boundary is used at the side of the FEM 
Table 1 Physical properties of duct

\begin{tabular}{cc}
\hline Material & Reinforced concrete \\
\hline Density & $2.4 \mathrm{t} / \mathrm{m}^{3}$ \\
\hline Young modulus & $2.65 \times 10^{7} \mathrm{kN} / \mathrm{m}^{2}$ \\
\hline Poisson ratio & 0.2 \\
\hline damping ratio & 0.05 \\
\hline
\end{tabular}

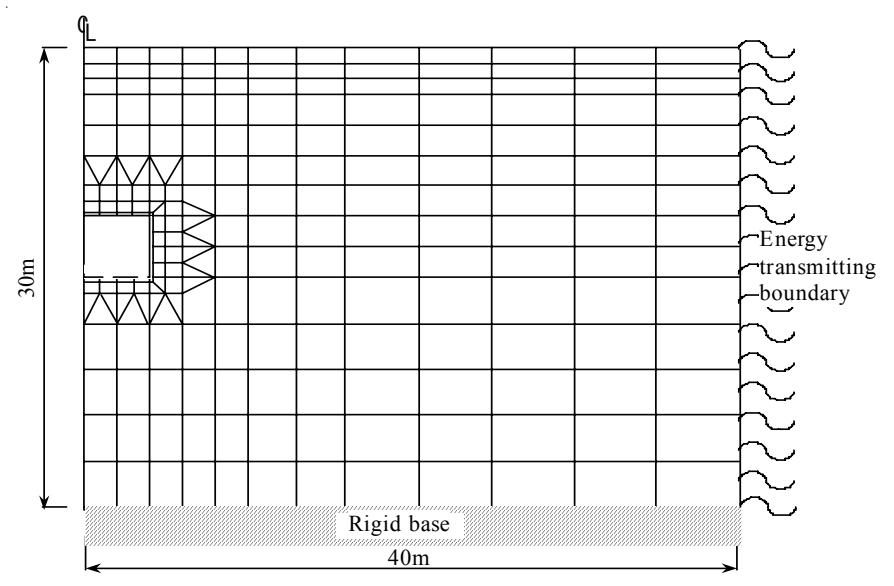

Fig. 5 Dynamic FEM model for covered duct model

Table 2 Physical properties of soil

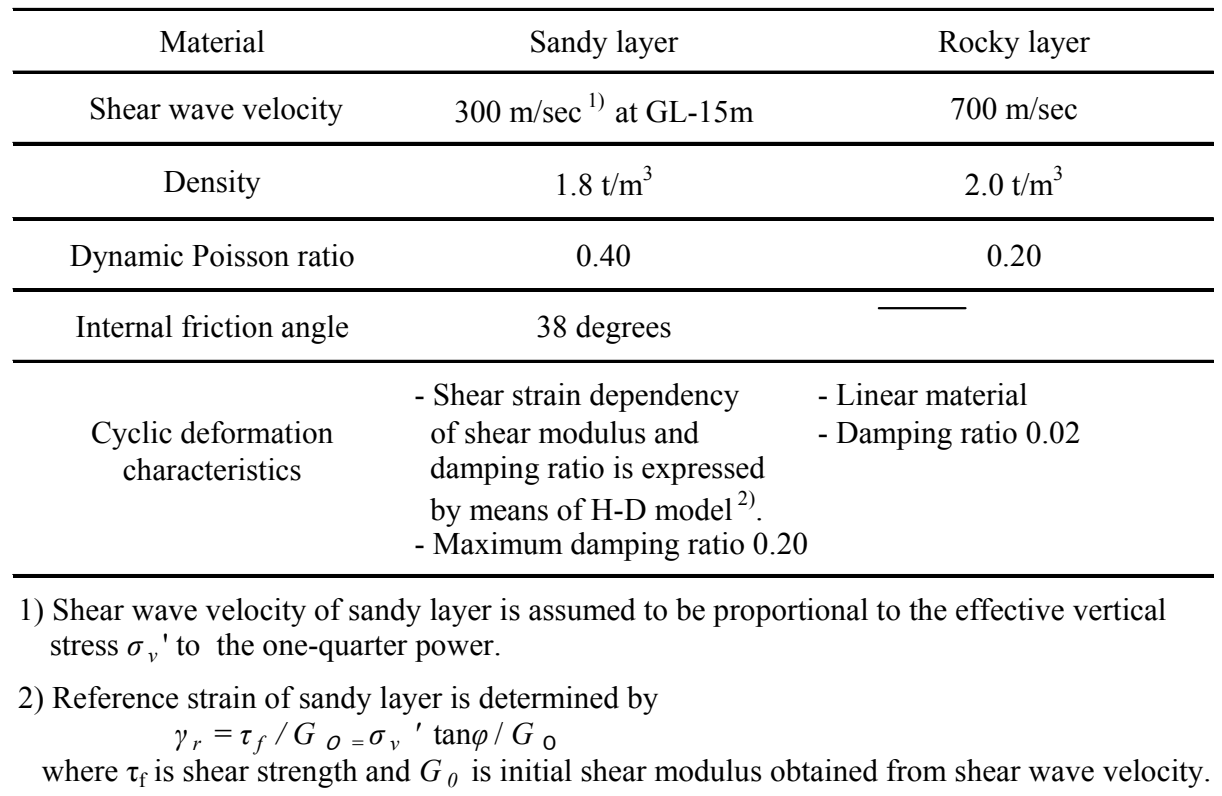

region and the bottom boundary is set to be a rigid base. Then, the response acceleration time history at a depth of GL-30m obtained from the multireflection analysis is input to the rigid base. The material properties of the duct and soil, in which the equivalent seismic rigidity of the layered soil is used, are treated as linear elements. The FEM model is shown in Fig. 5

Regarding FSDM, the relative displacement to the depth of GL-30m obtained from the multi-reflection analysis is applied to the both side boundaries of the FEM model as a forced displacement.

Regarding GRAMBS, the acceleration obtained from the multi-reflection analysis is applied to the ground and the duct.
For both FSDM and GRAMBS, the seismic loads are obtained from the response results at the same time of the multi-reflection analysis and the time is when maximum bending moment of the duct occurred in the dynamic FEM. This is because the objective of this study is to demonstrate the applicability of the seismic loads for the static seismic analysis methods of the soil-FEM type.

\section{(3) Numerical results}

The results of bending moments and axial forces for both the covered and uncovered duct models are shown in Fig. 6. The horizontal axis of the figure represents the unfolding coordinates in a roof slab, a 

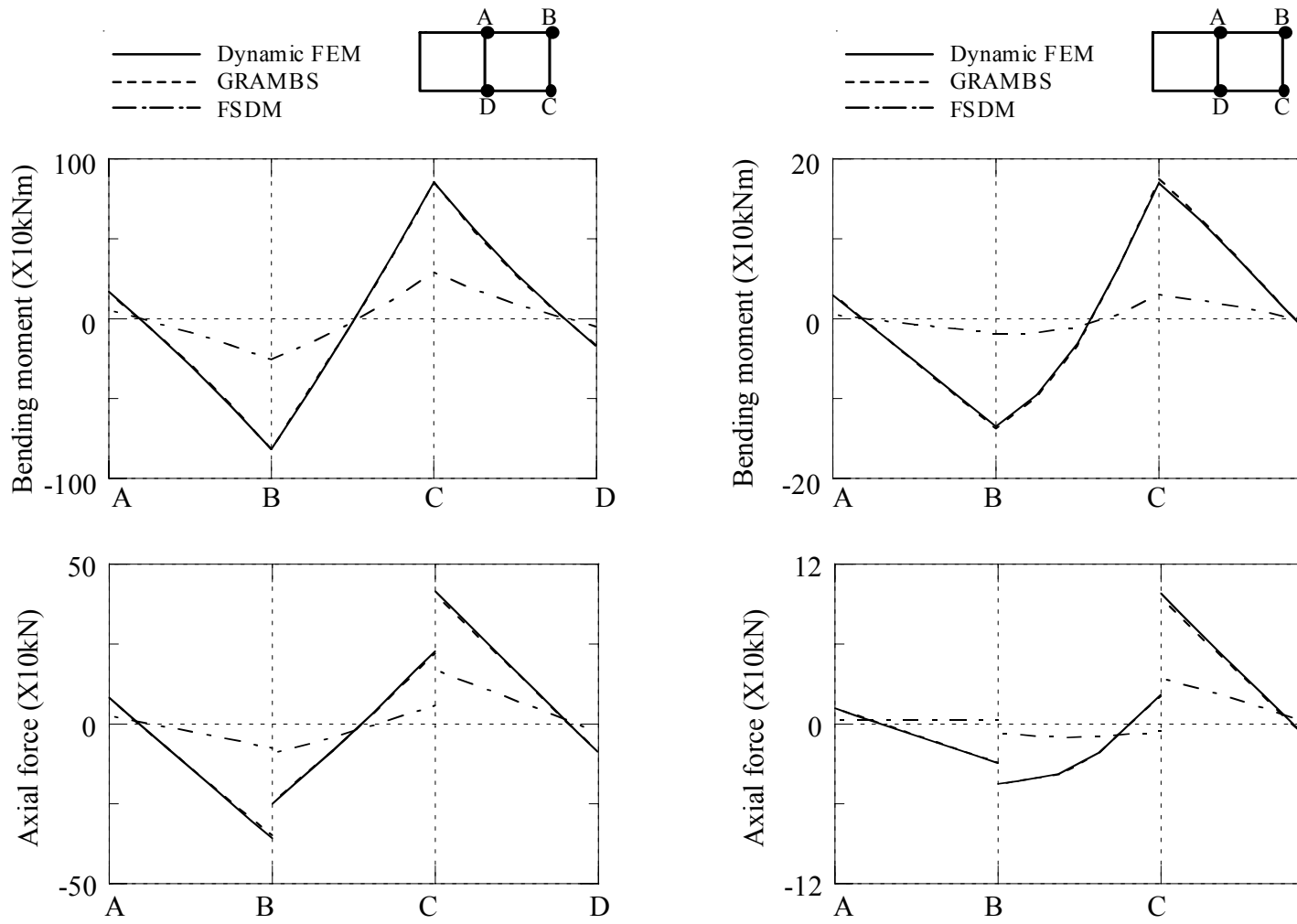

(a) Covered duct model
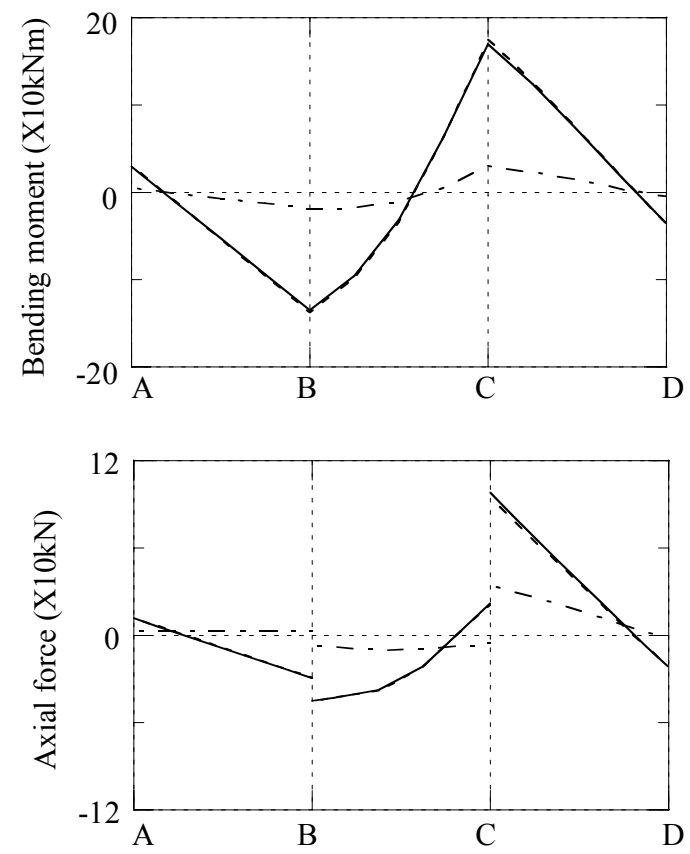

(b) Uncovered duct model

Fig. 6 Comparison of seismic increment sectional forces by dynamic FEM and existing static seismic analysis methods

side wall and a bottom slab. The following can be observed from the figures:

a) The bending moments and the axial forces of GRAMBS show good agreement with those of the dynamic FEM in both the models of the covered and uncovered duct models.

b) The bending moments of FSDM are $20 \%$ to $30 \%$ larger than those of the dynamic FEM in both models. In the case of the axial forces, the amplitudes are smaller and the distribution is different, when compared to the results of the dynamic FEM.

The results of the comparative analysis show the same tendency as those observed in the results that Hibino et al. ${ }^{9}$ achieved by means of the dynamic FEM, FSDM, and GRAMBS for a large underground rock cavern. That is, it is shown that GRAMBS yields almost the same responses as the dynamic FEM and that it is a very useful static seismic analysis method. On the other hand, FSDM yields smaller responses compared to dynamic FEM and it is an unsafe static seismic analysis method.

However, both the static seismic analysis methods are based on the characteristics of dynamic behavior of underground structures. Therefore, what kind of seismic load application is valid for underground structures in a static seismic analysis method of the soil-FEM type will be investigated.

\section{STATIC SEISMIC ANALYSIS BASED ON THE DYNAMIC SUBSTRUCTURE METHOD}

In this section, a new static seismic analysis method of the soil-FEM type for underground structures will be derived from the dynamic substructure method, which is an effective technique for analyzing a dynamic soil-structure interaction problem. The application of a seismic load is then studied.

\section{(1) Derivation of dynamic substructure method}

There are various types of dynamic substructure methods. The contact surface method adopted in this study was referred from a textbook ${ }^{7)}$ edited by JSCE. The details of the method are left to the papers $^{7), 8)}$, while the outline of the method is described hereafter.

The dynamic substructure method consists of the following 3 steps: 


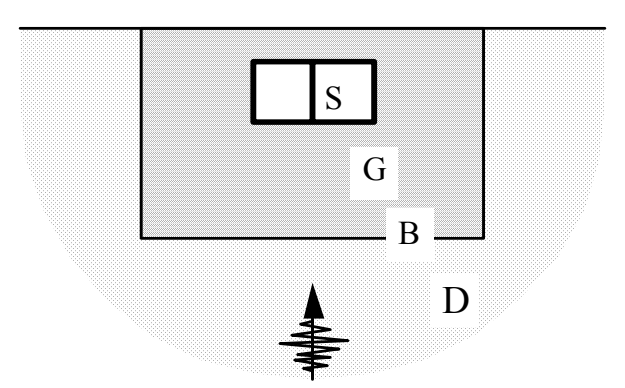

(a) Underground structure - near-field - far-field model

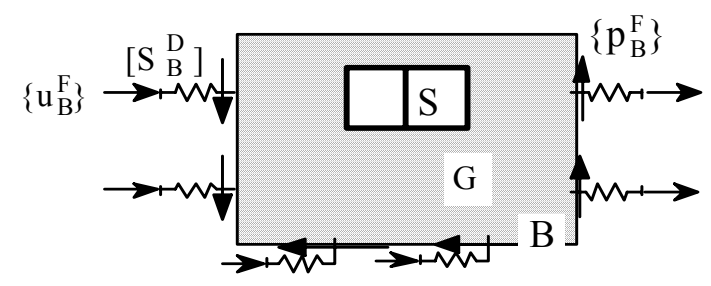

(b) Dynamic substructure method of underground structure and near-field

Fig. 7 Dynamic substructure method of soil-FEM type

\section{a) Step-1: input motion analysis}

An input motion corresponding to a seismic load in a general seismic design is obtained from a site response analysis in a free field or a ground from which an underground structure is removed (termed as a cavity field). Two-dimensional dynamic FEM should be used when a site response is calculated in a cavity field. A multi-reflection analysis can be conveniently used when using a free field. Thus, in this study, a free field is chosen to obtain a site response as an input motion. The input motions in a free field are the seismic displacements at the interfaces between a structure and the surrounding soil and the seismic forces equivalent to seismic stresses at the interfaces (termed as the equivalent seismic force).

\section{b) Step-2: ground impedance analysis}

Ground impedance corresponding to a subgrade reaction spring in a general seismic design is obtained from a cavity field.

\section{c) Step-3: structural analysis}

In the contact surface method, the ground impedances are attached to the outer surfaces of a substructure. A structural analysis is performed applying the input motion, and the seismic responses of the structure are then obtained.

Considering the objective of this study, the soil surrounding a structure is divided into a near-field and a far-field as shown in Fig. 7 and a substructure for this study is set to be the underground structure and the near-field. The dynamic substructure method is then applied to the dynamic interaction between the substructure and the far-field. The equilibrium equation of the dynamic substructure method in a frequency domain is as follows.

$$
\begin{array}{rcc}
{\left[\begin{array}{ccc}
{\left[S_{S S}\right]} & {\left[S_{S G}\right]} & \\
{\left[S_{G S}\right]} & {\left[S_{G G}\right]} & {\left[S_{G B}\right]} \\
& {\left[S_{B G}\right]} & {\left[S_{B B}\right]+\left[S_{B}^{D}\right]}
\end{array}\right]\left\{\begin{array}{l}
\left\{u_{S}\right\} \\
\left\{u_{G}\right\} \\
\left\{u_{B}\right\}
\end{array}\right\}} \\
=\left\{\begin{array}{c}
\{0\} \\
\{0\} \\
{\left[S_{B}^{D}\right]\left\{\begin{array}{c}
F \\
F
\end{array}\right\}+\left\{p_{B}^{F}\right\}}
\end{array}\right\}
\end{array}
$$

$[S]$ expresses the dynamic stiffness matrix of an underground structure or a near-field, and it is defined as

$$
[S]=-\omega^{2}[M]+i \omega[C]+[K]
$$

where $[M],[C]$, and $[K]$ denote mass matrix, damping matrix and stiffness matrix, respectively; $\omega$ is a predominant circular frequency; subscript $S$ denotes a structure; subscript $G$ denotes a near-field; subscript $B$ denotes a boundary between a near-field and a far-field; superscript $D$ denotes a cavity field; superscript $F$ denotes a free field; $\left[S_{B}^{D}\right]$ is a ground impedance obtained in the cavity field; and $\left\{u_{B}^{F}\right\}$ and $\left\{p_{B}^{F}\right\}$ are the seismic displacement and the excavation force which is an input motion in the free field, respectively.

\section{(2) Static substructure method of soil-FEM type}

A static seismic analysis method of the soil-FEM type for underground structures is derived from the equilibrium equation of the dynamic substructure method.

First, the equilibrium equation in a frequency domain is transformed into a time domain. The dynamic stiffness of an underground structure or a near-field is transformed based on Eq. (2). Although 
the ground impedance is usually a function of a frequency, it is assumed to be a frequency independent type of spring and dashpot in this study for the purpose of simplicity in derivation. Eq. (2) is rewritten as

$$
\left[S_{B}^{D}(\omega)\right]=\left[K_{B 0}^{D}\right]+i \omega\left[C_{B 1}^{D}\right]
$$

where $\left[K_{B 0}^{D}\right]$ and $\left[C_{B 1}^{D}\right]$ are the spring coefficient matrix and the damping coefficient matrix, respectively.

From Eq. (1) and Eq. (3), the equilibrium equation of the dynamic substructure method in time domain is expressed as

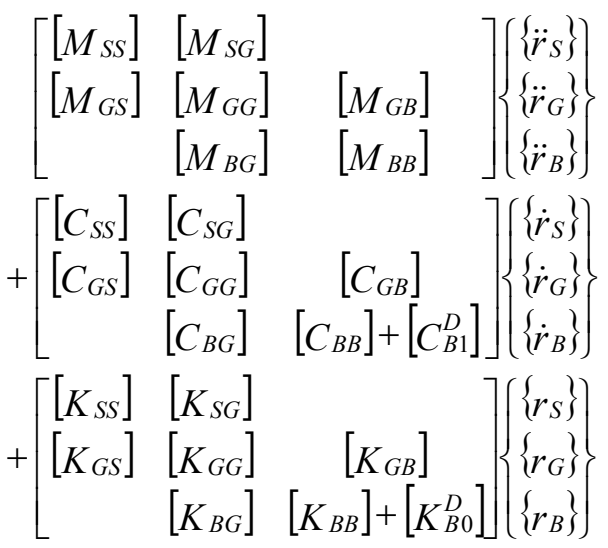

$$
\begin{aligned}
& =\left\{\begin{array}{c}
\{0\} \\
\{0\} \\
{\left[C_{B 1}^{D}\right]\left\{\dot{r}_{B}^{F}\right\}+\left[K_{B 0}^{D}\right]\left\{r_{B}^{F}\right\}+\left\{q_{B}^{F}\right\}}
\end{array}\right\}
\end{aligned}
$$

where a dot above a character denotes a derivative with respect to time, and $\{r\}$ and $\{q\}$ are a seismic displacement and the equivalent seismic force in time domain, respectively.

Second, the following assumptions are made in order to transform Eq. (4) into an equilibrium equation of a static seismic analysis method for underground structures.

Assumption 1:

A boundary of a substructure is set at a distance where an underground structure has little influence on displacements of the boundary.

Assumption 2:

A damping force is ignored.

Assumption 3:

Response accelerations of the structure and the near-field are the same as those of the free field at the same location.

Assumption 1 is aimed at simplifying a boundary technique. From this assumption, velocities and displacements at the boundary are approximately the same as those at the boundary of a free field, and thus the ground impedance is not necessary in Eq. (4).

Assumption 2 is valid because of the following explanation. The purpose of the main use of a static seismic analysis method is to calculate the maximum stresses of an underground structure. The maximum stresses generally occur when 1 st-mode shear deformation of the near-field occurs, so that velocities at 1st-mode shear deformation of the near-field are small and the damping force is also small at the mode. In addition, a damping force is generally small compared to an inertia force or a restoring force. By this assumption, the term related to the damping force can be neglected in Eq. (4).

Assumption 3 is valid because of the characteristics of dynamic behavior of underground structures described earlier. According to this assumption, the inertia forces in Eq. (4) can be replaced by the given seismic forces using the accelerations of the free field, and the replaced forces can be moved to the right-hand side.

With these three assumptions, the equilibrium equation of the dynamic substructure method in time domain is as follows:

$$
\begin{aligned}
& {\left[\begin{array}{lll}
{\left[K_{S S}\right]} & {\left[K_{S G}\right]} & \\
{\left[K_{G S}\right]} & {\left[K_{G G}\right]} & {\left[K_{G B}\right]} \\
& {\left[K_{B G}\right]} & {\left[K_{B B}\right]}
\end{array}\right]\left\{\begin{array}{l}
\left\{r_{S}\right\} \\
\left\{r_{G}\right\} \\
\left\{r_{B}\right\}
\end{array}\right\}}
\end{aligned}
$$

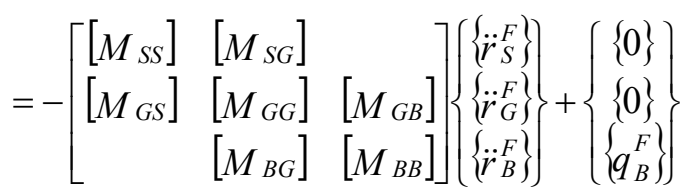

Eq. (5) has no unknown accelerations and no unknown velocities and it expresses a static analysis. In this study, the static seismic analysis method of the soil-FEM type expressed by Eq. (5) is termed Ground Response Method (GRM).

In GRM, inertia forces calculated using response accelerations of a free field are applied to a nearfield and an underground structure, and equivalent seismic forces calculated using response stresses of a free field are simultaneously applied to the outer boundaries of the substructure, as shown in Fig. 8.

A comparison of GRM and the existing static seismic analysis methods is made in the following. As shown in Fig. 2 and Fig. 8, GRM and GRAMBS are very similar, because both methods use inertia forces calculated using response accelerations of a free field as the main seismic load. However, the boundary conditions are different in the two methods. In GRAMBS, when a horizontal earthquake motion is input, the side boundaries use horizontal rollers and the bottom boundary uses a rigid base. 


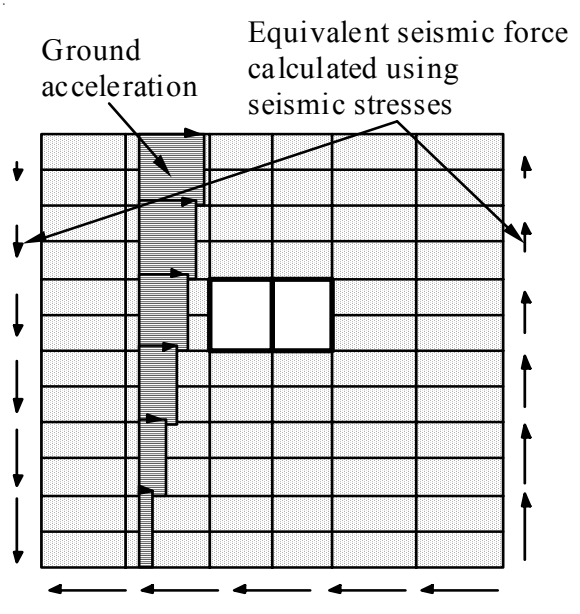

Fig .8 Seismic load of GRM

On the other hand, in GRM, equivalent seismic forces calculated using response stresses of a free field are applied to both the side boundaries and bottom boundary. However, if the assumption 1 is satisfied in the case of horizontal earthquake motion, the reaction forces at the horizontal roller of the side boundaries in GRAMBS are equal to the equivalent seismic forces at the side boundaries in GRM. Hence, both methods are equivalent to each other with respect to boundary conditions.

As shown in Fig. $\mathbf{1}$ and Fig. 8, it is clear that GRM and FSDM are different methods in terms of the main seismic forces. Although both methods use responses of a free field in order to calculate seismic deformation of an underground structure and a nearfield, GRM uses inertia forces of the near-field as the main seismic load, while FSDM uses seismic displacements of the outer boundaries of a near-field, and this difference is significant.

Since GRM is derived from the dynamic substructure method, it is valid as the static seismic analysis method of the soil-FEM type for underground structures as long as the three assumptions described earlier are satisfied. In the next sections, the limitations of FSDM are investigated, followed by the modification of FSDM.

\section{(3) Problem of FSDM and its modification ${ }^{11)}$}

In order to investigate the problem of the seismic load application in FSDM, both GRM and FSDM are performed using the two-dimensional FEM model of a free field, and the results are compared with that of the multi-reflection analysis. All the numerical conditions are the same as those described earlier.

As a result, seismic shear strain distributions of soil in the depth direction are shown in Fig. 9. The

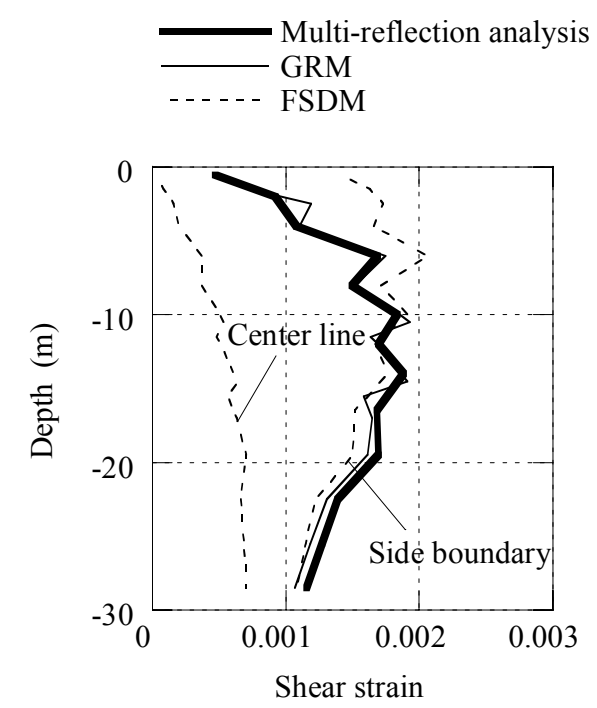

Fig. 9 Comparison of seismic strains of a free field

results of FSDM are shown at the center axis and at the side boundary, and those of GRM are only shown at the center axis. The following can be seen from the figures:

a) The shear strain distribution of GRM shows good agreement with that of the multi-reflection analysis. In addition, the distributions at every distance from the side boundary of GRM are the same.

b) The shear strain distribution at the side boundary of FSDM shows good agreement with that of the multi-reflection analysis, except in the vicinity of the ground surface. However, at the center axis, the amplitude of FSDM is $20 \%$ to $50 \%$ larger than that of the multi-reflection analysis, and there is a large difference between both distributions. This is because the forced displacements applied to the side boundary in FSDM cannot produce uniform shear deformation in the FEM model. However, in GRM, it can be produced by the inertia forces and the equivalent seismic rigidity.

Accordingly, it was found that GRM can reproduce shear deformation of a free field, but FSDM cannot reproduce it. Moreover, the shear strain distributions change at every distance from the side boundary at which the seismic displacement is applied. Therefore, the resulting seismic section forces of an underground structure are also definitely changed at every distance from the side boundary.

Hamada et al. ${ }^{3), 4)}$ demonstrated that the seismic deformation of an underground rock cavern is governed by the seismic strains of surrounding soil according to the record of earthquake motions at a mountain tunnel. They proposed that the forced displacement which is equivalent to the seismic strain be applied to the outer boundaries of an FEM model 


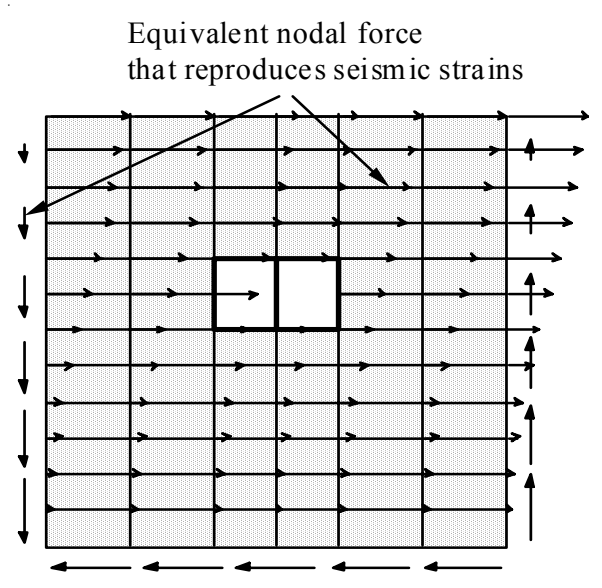

Fig. 10 Seismic load of modified-FSDM

as a static seismic analysis method for underground structures. However, in this study, it has been demonstrated that this seismic load application cannot reproduce the intended strains in the ground.

Therefore, equivalent nodal forces that theoretically reproduce seismic strains will be derived. First, a seismic stress $\left\{\sigma^{F}\right\}$ is obtained from the seismic strain $\left\{\varepsilon^{F}\right\}$ of a free field using Eq. (6), and then an equivalent nodal force $\left\{q^{F}\right\}$ is calculated from the seismic stress $\left\{\sigma^{F}\right\}$ using Eq. (7), i.e.,

$$
\begin{gathered}
\left\{\sigma_{e}^{F}\right\}=\left[D_{e}\right]\left\{\varepsilon_{e}^{F}\right\} \\
\left\{q^{F}\right\}=\sum_{e} \int_{v_{e}}\left[B_{e}\right]^{T}\left\{\sigma_{e}^{F}\right\} d V \\
=\sum_{e} \int_{v_{e}}\left[B_{e}\right]^{T}\left[D_{e}\right]\left\{\varepsilon_{e}^{F}\right\} d V
\end{gathered}
$$

where $[D]$ is the stress-strain matrix, $[B]$ is the strain-displacement matrix, and the subscript $e$ denotes a unit of the element.

Eq. (7) theoretically expresses the equivalent nodal forces that reproduce the seismic strains of a free field. The soil-FEM type of a static seismic analysis method using the equivalent seismic forces by Eq. (7) is termed as the modified FEM Seismic Deformation Method (modified-FSDM) in this study. The seismic load application of modifiedFSDM is shown in Fig. 10. It is confirmed that the same shear strain distribution as that of the multireflection analysis is reproduced at any distance from the side boundary when the derived seismic load is applied to the two-dimensional FEM model of a free field, the results of which are not presented in this paper.

Next, the relationship between the seismic load of modified-FSDM and GRM is studied.
First, since the relationship between a seismic strain $\left\{\varepsilon^{F}\right\}$ and an equivalent seismic displacement $\left\{r^{F}\right\}$ is obtained using Eq. (8), an equivalent nodal force $\left\{q^{F}\right\}$ is expressed by Eq. (9) and Eq. (10), i.e.,

$$
\begin{gathered}
\left\{\varepsilon_{e}^{F}\right\}=\left[B_{e}\right]\left\{r_{e}^{F}\right\} \\
\left\{q^{F}\right\}=[K]\left\{r^{F}\right\} \\
{[K]=\sum_{e} \int_{v_{e}}\left[B_{e}\right]^{T}\left[D_{e}\right]\left[B_{e}\right] d V}
\end{gathered}
$$

where $[K]$ is the stiffness matrix of soil.

Equation (9) shows that the equivalent nodal forces that reproduce seismic strains of a free field are obtained by multiplying the stiffness matrix of the soil and the seismic displacements of a free field.

On the other hand, the equilibrium equation of GRM for a free field is as follows:

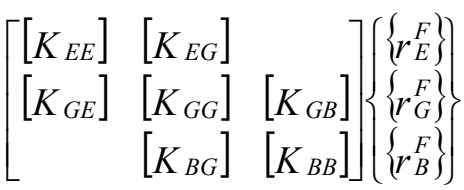

$$
\begin{aligned}
& =-\left[\begin{array}{lll}
{\left[M_{E E}\right]} & {\left[M_{E G}\right]} \\
{\left[M_{G E}\right]} & {\left[M_{G G}\right]} & {\left[M_{G B}\right]} \\
& {\left[M_{B G}\right]} & {\left[M_{B B}\right]}
\end{array}\right]\left\{\left\{\begin{array}{l}
\left\{\ddot{r}_{E}^{F}\right\} \\
\left\{\ddot{r}_{G}^{F}\right\} \\
\left\{\ddot{r}_{B}^{F}\right\}
\end{array}\right\}+\left\{\begin{array}{c}
\{0\} \\
\{0\} \\
\{F \\
\left.q_{B}^{F}\right\}
\end{array}\right\}\right.
\end{aligned}
$$

where subscript $E$ denotes an excavated soil for the construction of an underground structure.

Since the seismic load of the excavated soil is not necessary in Eq. (11), the rows of the degree of freedom $E$ in the stiffness and mass matrices are set to be zero, the columns of the degree of freedom $E$ are eliminated. Equation (11) is then transformed into

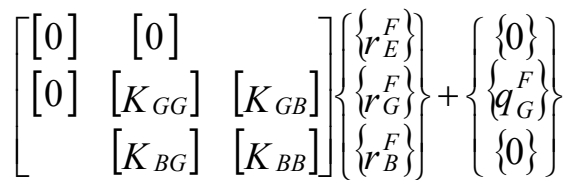

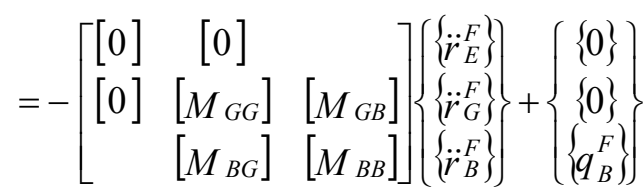

$$
\begin{aligned}
& \left\{q_{G}^{F}\right\}=\left[K_{G E}\right]\left\{r_{E}^{F}\right\}+\left[M_{G E}\right]\left\{\ddot{r}_{E}^{F}\right\}
\end{aligned}
$$



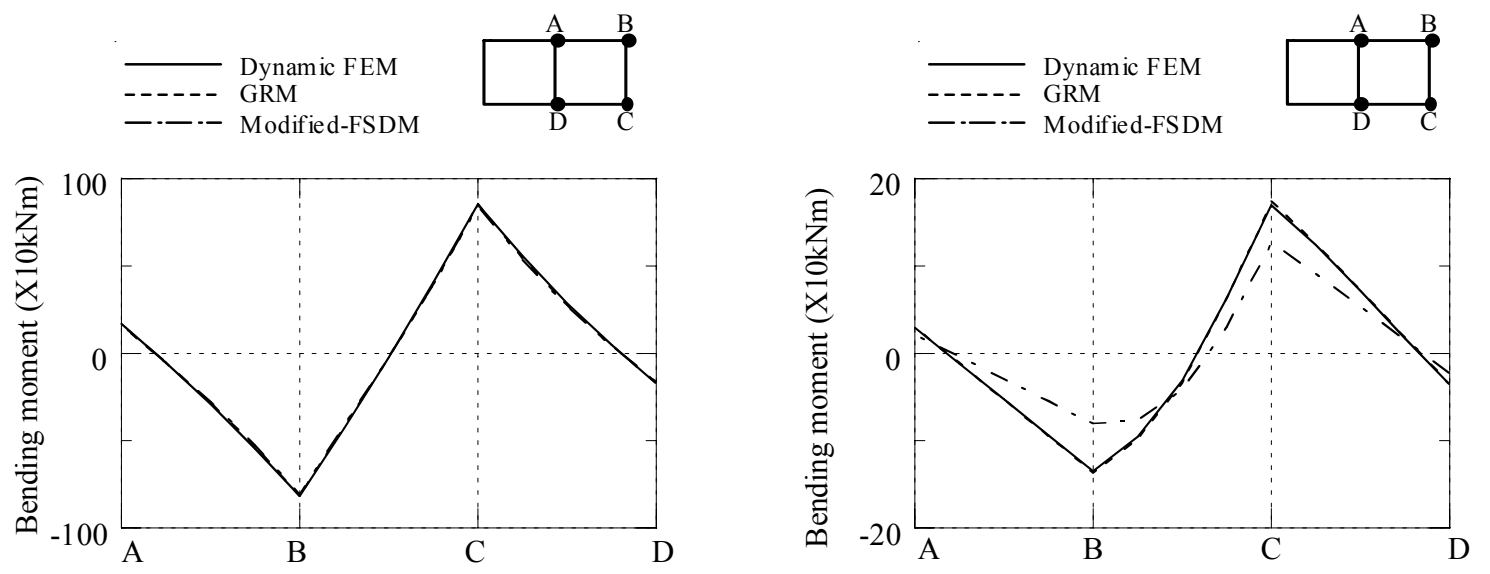

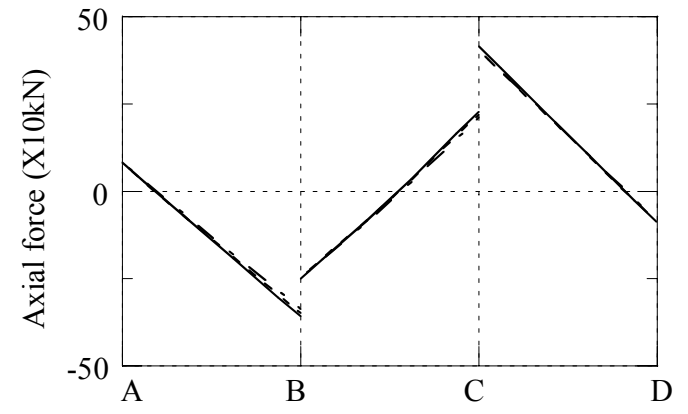

(a) Covered duct model

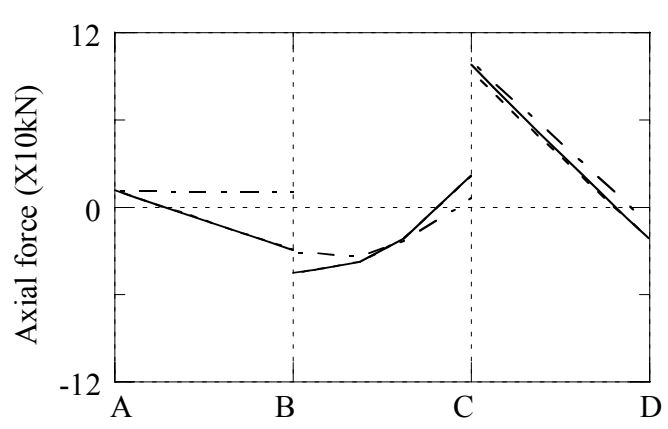

(b) Uncovered duct model

Fig. 11 Comparison of seismic increment sectional forces by dynamic FEM and proposed static seismic analysis methods

where $\left\{q_{G}^{F}\right\}$ denotes the seismic load that the excavated soil applies to the interfaces between the excavated soil and the near-field. This seismic load is equivalent to the seismic stresses at the interfaces, which are a kind of excavation force.

The left-hand side of Eq. (12) shows a multiplication between the stiffness matrix of a near-field and the seismic displacements of a free field. This expresses the seismic load of modified-FSDM referring to Eq. (9). The right-hand side of Eq. (12) is equal to the seismic load of GRM without the inertia forces of a structure, referring to Eq. (5). Consequently, it is clear that the seismic load of modifiedFSDM is equivalent to that of the seismic load of GRM without the inertia forces of a structure.

\section{(4) Verification analysis for an underground duct}

In order to verify the soil-FEM type of the static seismic analysis method proposed in this study, GRM and modified-FSDM were conducted for an underground duct in the transverse direction, and the results were compared with those of twodimensional dynamic FEM.

The model of the underground duct and the ground, the soil properties, the input earthquake motion, and the analytical method of dynamic FEM are the same as described earlier.
Regarding GRM, the response accelerations of a free field are applied to the near-field and the duct, and the equivalent seismic forces calculated using the response stresses of a free field are applied to the side boundaries.

Regarding modified-FSDM, the equivalent nodal forces that reproduce seismic strains of a free field are applied to the near-field, and the seismic load calculated using the response stresses of an excavated soil are applied to the interfaces between the duct and the near-field.

The seismic loads for both the static analyses are obtained from the multi-reflection analysis at the time when maximum bending moment of the duct occurred in the dynamic FEM.

The results are shown in the figures of bending moments and axial forces for both the covered and uncovered duct models in Fig. 11. The horizontal axis of the figure represents the unfolding coordinates in a roof slab, a side wall and a bottom slab. The following can be observed from the figures:

a) The bending moments and the axial forces of GRM show good agreement with those of the dynamic FEM in the cases of both the covered and uncovered duct models.

b) The bending moments and axial forces of the modified-FSDM show good agreement with 
Table 3 Seismic load application for static seismic analysis method of soil-FEM type

\begin{tabular}{|c|c|c|c|}
\hline & Existing method & & Proposed method \\
\hline \multirow[t]{2}{*}{ GRAMBS } & $\begin{array}{l}\text { - Inertia forces calculated using } \\
\text { response accelerations of a free field } \\
\text { to underground structures and } \\
\text { a near-field }\end{array}$ & \multirow[t]{2}{*}{ GRM } & $\begin{array}{l}\text { - Inertia forces calculated using } \\
\text { response accelerations of a free field } \\
\text { to underground structures and } \\
\text { a near-field }\end{array}$ \\
\hline & $\begin{array}{l}\text { - Side boundary is } \\
\text { S-wave input; horizontal roller } \\
\text { P-wave input; vertical roller }\end{array}$ & & $\begin{array}{l}\text { - Equivalent seismic forces calculated } \\
\text { using seismic stresses of a free field } \\
\text { to outer boundaries of a near-field }\end{array}$ \\
\hline \multirow[t]{2}{*}{ FSDM } & $\begin{array}{l}\text { - Forced displacements calculated using } \\
\text { response displacements of a free field } \\
\text { to side boundaries of a near-field }\end{array}$ & \multirow{2}{*}{$\begin{array}{l}\text { Modified } \\
\text { FSDM }\end{array}$} & $\begin{array}{l}\text { - Equivalent nodal forces that reproduce } \\
\text { seismic strains of a free field } \\
\text { to a near-field }\end{array}$ \\
\hline & & & $\begin{array}{l}\text { - Equivalent seismic forces calculated } \\
\text { using seismic stresses of an excavated } \\
\text { ground to interfaces between structures } \\
\text { and a near-field }\end{array}$ \\
\hline
\end{tabular}

those of the dynamic FEM in the case of the covered duct model. However, in the case of the uncovered duct model, the bending moments are $60 \%$ to $70 \%$ larger than those of the dynamic FEM, and the distribution of the axial forces is different from that of the dynamic FEM. This is because the inertia forces of the duct are not applied in modified-FSDM. It is confirmed that, in the case of the uncovered duct model, the section forces of modifiedFSDM show good agreement with those of GRM without the inertia forces of the duct, whose results are not presented in this paper.

Consequently, it has been found that GRM gives almost the same responses as the dynamic FEM and that it is an alternative seismic analysis method for underground structures, which can be used instead of the dynamic FEM. On the other hand, the responses of modified-FSDM are different in the case of uncovered underground structures in which the inertia forces of a structure have a large influence on the responses; however, it yields almost the same responses as the dynamic FEM in the case of ordinary covered underground structures.

\section{CHARACTERISTICS OF SOIL-FEM TYPE OF STATIC SEISMIC ANALYSIS METHOD}

The characteristics of the soil-FEM type of a static seismic analysis method for underground structures studied in the previous section will be made clear, and the generality of the static seismic analysis method will be described.

The seismic load applications of the existing static seismic analysis methods and the proposed static seismic analysis methods in this study are shown in Table 3.

GRM is derived from the dynamic substructure method assuming three conditions for underground structures. Essentially, there are no limitations on the shape and dimensions of a substructure as an object of study in the dynamic substructure method. There are also no limitations on the kind of input earthquake motion. Thus, as long as the three assumptions are satisfied, GRM is applicable to any type of underground structures and any kind of input earthquake motions based on the derivation. That is, regarding the type of an underground structure, GRM is applicable to an underground structure with a very wide cross section such as an underground parking structure, as well as to a cylindrical underground structure such as an underground tank. With regard to the kind of input earthquake motion, GRM is applicable to a longitudinal wave input, a surface wave input, and an inclined input of a shear wave or longitudinal wave.

Moreover, a characteristic of GRM is that it is not necessary to prepare an FEM model up to the ground surface in modeling a structure and a nearfield as a substructure. That is, when a part of the ground without the ground surface is extracted, equivalent seismic forces calculated using the response stresses of a free field are applied to the up- 


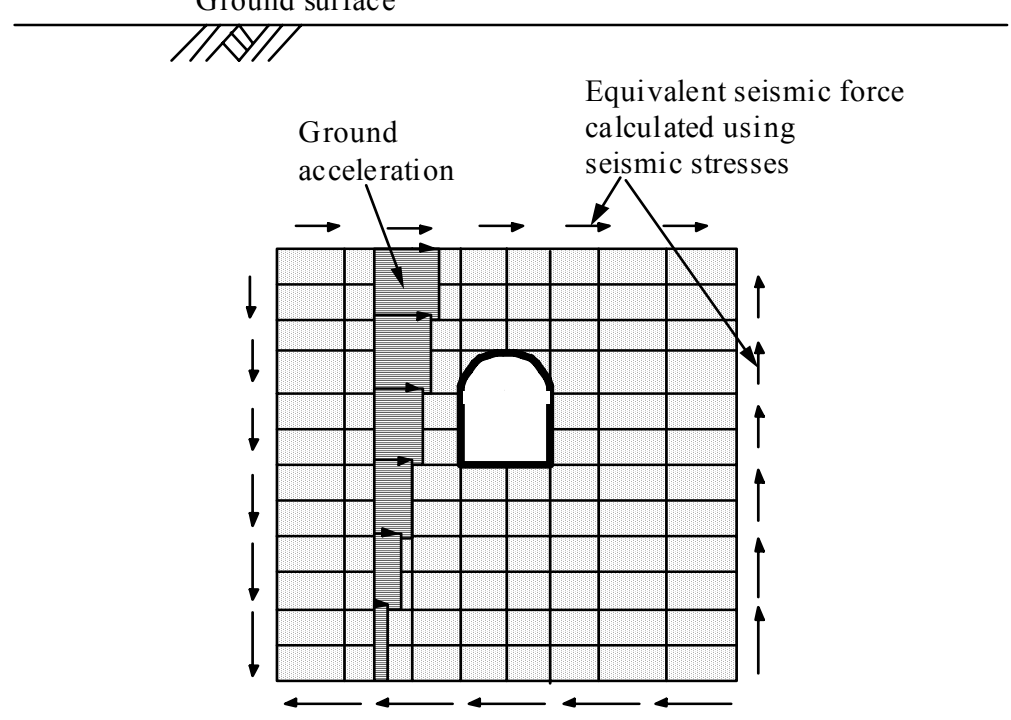

Fig. 12 Seismic analysis of very deep underground cavern by means of GRM

per boundary as well as the other boundaries. This characteristic is very effective for the seismic design of a very deep underground cavern as shown in Fig. 12. In the case that the outer boundaries are set at the distance of $2 D(D$, the average value of the width and the height of a cavern) from the cavern, the seismic stresses of the covered concrete of GRM show good agreement with those of the dynamic FEM $^{12)}$.

Regarding GRAMBS, the main seismic load is inertia forces calculated using response accelerations of a free field, and the resulting reaction forces at side and bottom boundaries are anticipated to be equal to the equivalent seismic forces calculated using seismic stresses for GRM, in the case of a vertical input of shear wave or longitudinal wave. Thus, it is concluded that GRAMBS is equivalent to GRM in the case of a vertical input and that there are no limitations on the shape and dimensions of an underground structure as in GRM. However, GRAMBS is not applicable to an inclined input of a shear wave and a longitudinal wave because of constraints of displacements at the side and bottom boundaries. In addition, considering the necessity of modeling up to the ground level, GRAMBS is inferior to GRM with respect to the generality of the static seismic analysis method. However, since it is not necessary to consider these numerical conditions in a common seismic design of underground structures, there is no doubt that GRAMBS is one of the most effective static seismic analysis methods.

Regarding the modified-FSDM, the seismic load is theoretically equivalent to that of GRM except for the inertia forces of an underground structure. Thus, it is concluded that modified-FSDM is practically equivalent to GRM when the inertia forces of an underground structure have little influence, and there are no limitations on the shape and dimensions of an underground structure as in GRM. Moreover, if seismic strains or seismic stresses of a free field are calculated, there are also no limitations on the kind of input wave and there is no need to prepare an FEM model up to the ground surface. Accordingly, considering the influence on inertia forces of a structure and the function of the analysis program used, modified-FSDM is an alternative static seismic analysis method instead of GRM.

Regarding FSDM, seismic strains of a free field cannot be reproduced, and the resulting seismic section forces of an underground structure are different at various distances from the side boundary; thus FSDM cannot be recommended.

\section{CONCLUSIONS}

With regard to the soil-FEM type of static seismic analysis methods, in which a near-field of an underground structure is modeled using finite elements, the author has demonstrated the seismic load application based on the dynamic substructure method and has verified its validity through the numerical analyses of the underground duct in the transverse direction. The conclusions are as follows:

(1) The soil-FEM type of a static substructure method for underground structures, GRM, is derived from the dynamic substructure method, with an assumption based on the characteristics of dynamic behavior of underground structures and another assumption aimed at simplifying the bound- 
ary technique. In GRM, inertia forces calculated using response accelerations of a free field are applied to a near-field and an underground structure, and equivalent seismic forces calculated using response stresses of a free field are simultaneously applied to the outer boundaries of the substructure.

(2) Regarding the existing static seismic analysis methods, GRAMBS is a method very similar to GRM. This is because the main seismic load for both methods is inertia force calculated using the response accelerations of a free field and this seismic load produces seismic deformation of a nearfield. In a common seismic design of underground structures, GRAMBS is equivalent to GRM. On the other hand, FSDM is quite a different method from GRM, because seismic deformation of a near-field is produced by applying seismic displacements to outer boundaries of a near-field.

(3) Since it is confirmed through the numerical analyses that FSDM cannot reproduce seismic strains of a near-field, modified-FSDM is proposed, in which equivalent nodal forces that reproduce seismic strains of a free field are applied to a nearfield. It has been demonstrated that the seismic load of modified-FSDM is theoretically equivalent to that of GRM except in the case of inertia forces of an underground structure.

(4) The advantages of the methods proposed in this study, GRM and modified-FSDM, are that there is no need to prepare an FEM model up to the ground surface and there are no limitations on the kind of input wave, because there are no displacement constraints on the outer boundaries of a near-field. Further, the proposed methods are more general than the existing static seismic analysis methods.

\section{FUTURE RESEARCH}

The construction of large important underground structures such as a large underground tank and an underground nuclear power plant is planned, and those structures are required to be extremely safe against earthquakes.

The soil-FEM type of static seismic analysis method, in place of the dynamic FEM, is applicable to the seismic design of these important underground structures and is very effective in parameter studies of basic design steps and detailed design steps.

In the future, the author will study the applicability of GRM to a seismic design of large underground structures such as a very wide cross section underground structure and a large cylindrical underground structure.

\section{REFERENCES}

1) The Ministry of Construction: Establishment of New Aseismic Design Method, 1977. (In Japanese)

2) Committee of Civil Engineering of Nuclear Power Facilities of JSCE: Evaluation method of investigation and testing methods for geology and soil in nuclear power facilities and seismic stability of soils and foundations - 6 Evaluation method of seismic safety for civil engineering underground structures, 1985. (In Japanese)

3) Hamada, M., Sugihara, Y., Shiba, Y. and Iwano, M.: Observation and study on dynamic behavior of rock cavern during earthquake, J. of JSCE, No. 341, pp. 187-196, 1984. (In Japanese)

4) Hamada, M., Izumi, H., Iwano, M. and Shiba, Y.: Analysis of dynamic strain around rock cavern and earthquake resistant design, J. of JSCE, No. 341, pp. 197-205, 1984. (In Japanese)

5) Katayama, I., Adachi, M., Shimada, Y., Tsuzuki, T. and Seshita, Y.: Proposal of practical quasi-dynamic analysis method for underground structures named Ground Response Acceleration Method for Buried Structures, Proc. of the 40th annual conference of JSCE, Vol. 1, pp. 737-738, 1985. (In Japanese)

6) Katayama, I.: Studies on fundamental problems in seismic design analyses of critical structures and facilities, Chap. 4, pp. 188-237, a doctoral thesis of Kyoto University, 1990.

7) JSCE: Dynamic analysis and seismic design - Vol. 2 Dynamic analysis method, GIHODO SHUPPAN, 1989. (In Japanese)

8) Tateishi, A.: A study on loading method of seismic deformation method, J. of JSCE, No. 441/I-18, pp. 157-166, 1992. (In Japanese)

9) Hibino, S., Komada, H., Honsho, S., Fujiwara, Y., Motojima, M., Nakagawa, K. and Nosaki, T.: Case study of siting technology for underground nuclear power plant, CRIEPI Report No. U17, 1991. (In Japanese)

10) Tateishi, A. and Sakashita, K.: Seismic analysis method of underground structures by means of static FEM based on dynamic substructure method, Proc. of the 47th annual conference of JSCE, Vol. 1, pp. 1466-1467, 1992. (In Japanese)

11) Tateishi, A. and Sakashita, K.: A study on seismic analysis method of underground structures by means of static FEM, Proc. of the 21st JSCE Earthquake Engineering Conference, pp. 469-472, 1991. (In Japanese)

12) Tateishi, A., Ueda, T. and Izumi, H.: Static seismic analysis method for deep rock cavern, Proc. of the 29th Japan national conference on soil mechanics and foundation engineering, pp. 1079-1080, 1994. (In Japanese)

(Received October 4, 2004) 